\title{
Elektrische Energietechnik - Der Wandel zu hybriden AC/DC-Netzen
}

\author{
U. Schichler OVE
}

Online publiziert am 12. November 2020

(c) Springer-Verlag GmbH Austria, ein Teil von Springer Nature 2020

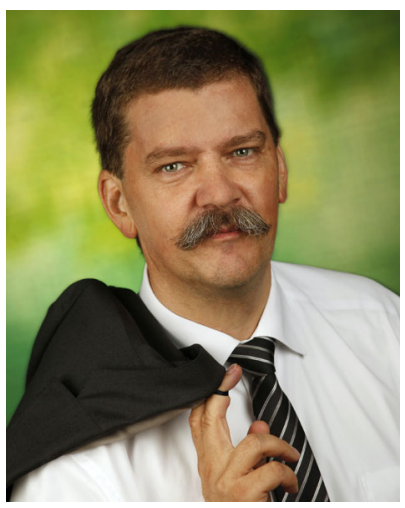

Univ.-Prof. Dr.-Ing. Uwe Schichler
Die international vereinbarten Klimaschutzziele und die damit verbundene notwendige Dekarbonisierung der Gesellschaft haben in Form der international stattfindenden Energiewende deutliche Auswirkungen auf die elektrische Energietechnik. Aktuelle Innovationen sind unter anderem im Bereich der Entwicklung und Anwendung der Gleichstromtechnologie in der Nieder-, Mittel- und Hochspannungsebene und der zunehmenden Integration von leistungselektronisch mit dem Netz gekoppelten Energieerzeugern zu finden. Dabei werden zahlreiche Innovationsbeiträge von österreichischen Universitäten und Forschungsinstitutionen in Kooperationen mit Industrie- und Wirtschaftsunternehmen sowie durch geförderte Forschungsprojekte erbracht. Das Ziel der Forschungsarbeiten ist es, eine zuverlässige und sichere Versorgung der Gesellschaft mit elektrischer Energie in Verbindung mit einer $\mathrm{CO}_{2}$ Reduktion zu realisieren.

Die vorliegende e\&i-Ausgabe gibt einen ausgewählten Überblick zur Anwendung der Gleichstromtechnologie in hybriden AC/DCNetzen und zu den aktuellen Herausforderungen in der elektrischen Energietechnik.

Die erste Arbeit der vorliegenden Beiträge liegt thematisch im Bereich der Hochspannungs-Gleichstromübertragung (HGÜ) und beschreibt aus der Sicht eines Übertragungsnetzbetreibers die Planungen zur Vorbereitung zukünftiger leistungselektronisch geprägter Energiesysteme und für die Implementierung von MultiterminalFunktionalitäten in hybriden AC/DC-Systemen in Deutschland und Europa. Bestehende Systemanforderungen und die Möglichkeiten zur Eingliederung von Multiterminal-HGÜ-Overlay-Netzen werden beschrieben. Weiterführende Forschungsaktivitäten zu technischen Fragestellungen rund um den sicheren und zuverlässigen Betrieb von leistungselektronisch dominierten Systemen werden dargestellt. Beispielhaft werden Simulationsergebnisse für Multiterminal-HGÜSysteme als Planungsoption diskutiert.

Der zweite Artikel beschreibt ein erweitertes Regelkonzept für eingebettete VSC-HGÜ-Leitungen mit dem Fokus auf einer Stützung des parallelen AC-Systems nach Störungen wie Leitungs- und Generatorausfällen. Die Betriebsziele der Regelung bestehen einerseits darin, die direkten Auswirkungen der Störung auf das AC-System zu minimieren, und andererseits die Sicherstellung einer langfristigen Spannungsstabilität. Das vorgestellte Regelkonzept wird in einem Testsystem mit einfacher Netztopologie untersucht und seine
Effektivität mit der von bereits vorhandenen Regelstrategien verglichen. Im Testsystem sind das erforderliche dynamische Verhalten der VSC-Umrichter, die Betriebsgrenzen der VSC-HGÜ-Leitung sowie ein für Spannungsstabilitätsbetrachtungen geeignetes dynamisches Lastverhalten implementiert. Die Simulationsergebnisse werden hinsichtlich der Funktionsweise und der sich daraus ergebenden Vorteile des vorgestellten Regelkonzeptes diskutiert.

Im dritten Beitrag werden derzeit laufende Forschungsaktivitäten auf dem Gebiet der geomagnetisch induzierten Ströme (GIC) thematisiert. Die Auswirkungen von GIC auf Transformatoren sind unter anderem ein erhöhter Scheinleistungsbedarf und eine daraus resultierende verringerte Belastbarkeit. Diese Arbeit zeigt, wie geomagnetisch induzierte Ströme innerhalb eines Übertragungsnetzes mit geerdeten Transformatorsternpunkten berechnet werden können. Für die Berechnung werden global verfügbare Magnetfelddaten und ein globales dreidimensionales Leitfähigkeitsmodell der Erde benutzt. Durch die Überlagerung der GIC aller Freileitungen, die an einen Transformator angeschlossen sind, kann der resultierende geomagnetisch induzierte Strom ermittelt werden. Die berechneten GIC-Ströme werden mit Messungen von Transformatorsternpunkten verglichen.

Der vierte Beitrag beschreibt das österreichische Forschungsprojekt „ADC Labs", bei dem die Grundlagen für Testmethoden zur Unterstützung des Entwicklungs- und Validierungsprozesses von DCKomponenten und -Systemen auf Basis von Power-Hardware-inthe-Loop (P-HIL) erforscht wurden. Dabei wurden Synergien zwischen den Institutionen TU Graz (Hochspannung) und AIT (Niederund Mittelspannung) genutzt. Es wird im Detail auf die Entwicklung von Testmethoden und Forschungsinfrastruktur für Mittel- und Niederspannungs-DC-Systeme eingegangen.

Im abschließenden fünften Bericht wird Bezug genommen auf leistungselektronisch gekoppelte Windkraftwerke (WKW). Die Auslegung der elektrischen Betriebsmittel sowie die Planung und der Betrieb der Netze muss zunehmend an den Eigenschaften solcher Anlagen ausgerichtet werden. Wegen ihrer technischen Komplexität stellen dabei WKW mit doppelt speisenden Generatoren eine besondere Herausforderung dar, da bei innen sowohl die physikalischen Eigenschaften der rotierenden elektrischen Maschinen als auch das maßgeblich durch Steuerung und Regelung beeinflusste Verhalten des Umrichters zum Tragen kommen. Es wird das Verhalten der aktuellen Technologie von DFIG bei Kurzschlüssen und anderen Netzfehlern beschrieben, der Stand der Technik in der für die Anlagenauslegung wichtigen Kurzschlussnorm erläutert sowie ein neuer Vorschlag für die Nachbildung der WKW mit DFIG in dieser Norm unterbreitet.

Schichler, Uwe, Institut für Hochspannungstechnik und Systemmanagement, Technische Universität Graz, Inffeldgasse 18, 8010, Graz, Österreich (E-Mail: uwe.schichler@tugraz.at) 
Ich bedanke mich bei allen Autor/innen für die sorgfältige Erstellung der Beiträge und bei den Reviewern sowie der Redaktion für die kritische und sorgfältige Durchsicht der Originalarbeiten. Ich wünsche Ihnen, liebe Leserinnen und Leser, eine interessante Lektüre der vorliegenden Beiträge.
Hinweis des Verlags Der Verlag bleibt in Hinblick auf geografische Zuordnungen und Gebietsbezeichnungen in veröffentlichten Karten und Institutsadressen neutral. 